Accepted Manuscript

Hillock-free and atomically smooth InSb QWs grown on GaAs substrates by MBE

Y. Shi, E. Bergeron, F. Sfigakis, J. Baugh, Z.R. Wasilewski

PII:

S0022-0248(19)30119-8

DOI: https://doi.org/10.1016/j.jcrysgro.2019.02.039

Reference:

CRYS 24993

To appear in:

Journal of Crystal Growth

Revised Date: $\quad 6$ February 2019

Accepted Date: $\quad 15$ February 2019

Please cite this article as: Y. Shi, E. Bergeron, F. Sfigakis, J. Baugh, Z.R. Wasilewski, Hillock-free and atomically smooth InSb QWs grown on GaAs substrates by MBE, Journal of Crystal Growth (2019), doi: https://doi.org/ 10.1016/j.jcrysgro.2019.02.039

This is a PDF file of an unedited manuscript that has been accepted for publication. As a service to our customers we are providing this early version of the manuscript. The manuscript will undergo copyediting, typesetting, and review of the resulting proof before it is published in its final form. Please note that during the production process errors may be discovered which could affect the content, and all legal disclaimers that apply to the journal pertain.

The final publication is available at Elsevier via https://doi.org/10.1016/j.jcrysgro.2019.02.039. @ 2019. This manuscript version is made available under the CC-BY-NC-ND 4.0 license http://creativecommons.org/licenses/by-nc-nd/4.0/ 


\title{
Hillock-free and atomically smooth InSb QWs grown on GaAs substrates by MBE
}

\author{
Y. Shi ${ }^{\mathrm{a}}$, E. Bergeron ${ }^{\mathrm{a}, \mathrm{d}}$, F. Sfigakis ${ }^{\mathrm{c}, \mathrm{d}}$, J. Baugh $^{\mathrm{a}, \mathrm{c}, \mathrm{d}}$, Z.R. Wasilewski ${ }^{\mathrm{a}, \mathrm{b}, \mathrm{e}, *}$ \\ ${ }^{a}$ Department of Physics and Astronomy \\ ${ }^{b}$ Department of Electrical and Computer Engineering \\ ${ }^{c}$ Department of Chemistry \\ ${ }^{d}$ Institute for Quantum Computing (IQC) \\ ${ }^{e}$ Waterloo Institute for Nanotechnology (WIN) \\ University of Waterloo, 200 University Ave. W, Waterloo, ON, N2L 3G1, Canada
}

\begin{abstract}
Comprehensive studies on the surface morphological evolution of AlInSb metamorphic buffers and InSb QWs grown on top were conducted as a function of the GaAs (001) substrate offcut angles. We confirmed our earlier postulation that the vicinal surfaces defined by the hillock facets have the exact surface orientation needed to achieve large-area hillock-free surfaces. The related morphological transitions were discussed with a graphic illustration. The optimum substrate offcut for InSb towards [1110] direction was found to be around $0.5 \sim 0.6^{\circ}$ with our growth conditions. On 2-inch GaAs (001) substrates with this offcut, a hillock-free and atomically smooth surface morphology was successfully achieved for modulation-doped InSb QWs.
\end{abstract}

Keywords: A1. Surface morphology, A1. Substrate offcut, A1. Spiral growth, A3. InSb QW, A3. Metamorphic buffer

\section{Introduction}

InSb has the smallest effective mass, the largest Land g-factor and a strong spin-orbit coupling among all the binary III-V semiconductors, which makes it one of the most desirable material for realizing Majorana Zero modes (MZM) for topological quantum computing. [1-4] Several devices fabricated with

5 self-assembled InSb nanowires have been implemented, strongly suggesting the existence of MZMs. [5-8] Yet the top-down fabrication from a 2D electron gas (2DEG) is a much more scalable and cleaner approach. $[4,9] \mathrm{By}$ applying negative top gates, the area where the 2DEG is uncovered by the superconducting layer can be depleted, leaving only 1D conducting channels strongly coupled to the superconducting material. This motivates a thorough investigation on the high-quality InSb quantum wells (QW) grown by molecular beam epitaxy (MBE). Due to the lack of semi-insulating InSb substrates, such QWs are typically grown on high-quality GaAs substrates, with well-designed AlInSb metamorphic buffers to bring the lattice constant to that of InSb and minimize the propagating threading dislocations (TD). [10-13] To our best knowledge, the lowest threading dislocation density (TDD) has been achieved for InSb QWs grown on GaAs substrates so far is around $10^{8} \mathrm{~cm}^{-2},[10,13,14]$, which is still quite far below the world's expectation for QW devices, but tolerable for the top-down fabrication of nanowires. In fact, we have demonstrated that we can easily

\footnotetext{
${ }^{*}$ Corresponding author

Email address: zbig.wasilewski@uwaterloo.ca (Z.R. Wasilewski)
} 
find up to $1 \mu \mathrm{m}^{2}$ and TD-free areas on the surface of our AlInSb metamorphic buffer. [14] Further reduction of TDD is definitely indispensable and is currently under our investigation. This paper will focus on the improvement of surface morphology for InSb QWs.

As a result of high TDD, a high density of pyramid-shaped hillocks form on the surface of the AlInSb metamorphic buffers and InSb QWs, due to the spiral growth around the screw components of TDs, as predicted in 1951 by the BCF theory. [15] These hillocks don't seem to pose any serious issues on the first sight, as their sidewalls are atomically smooth and their facet angles are less than $1^{\circ}$. [14] However, the different facet angles in [1110] and [110] directions indicate possibly dissimilar probabilities for the attachment of $\mathrm{Al}$ and In atoms on hillock sidewalls along different directions, which may cause non-uniformity in AlInSb barrier composition, [16, 17] giving rise to fluctuations in confining potentials and local strains. The low-temperature mobility measured with InSb QW structures have always been far from ideal comparing to behavior of InSb bulk materials (which has the highest mobility of any III-V semiconductor at room temperature), and it has been suggested lately that the reason might be related to surface hillocks, with the boundaries of which acting as scattering centres. [18] Therefore, suppression of hillocks is essential from ( Recently we proposed that the vicinal surfaces defined by the naturally formed hillock facets have the exact surface orientation needed to achieve large-area hillock-free surfaces. [14] Here we report a comparative study on the surface morphology with and without an InSb QW on top of an AlInSb metamorphic buffer, as a function of substrate offcut angle. With the suitable offcut found in this study, we present a hillock-free and atomically 35 smooth InSb QW grown on a GaAs substrate.

\section{Experiment}

The modulation-doped InSb QWs and AlInSb metamorphic buffers were grown on 2-inch semi-insulating GaAs (001) substrates using a Veeco Gen10 MBE system. A schematic representation of the structures is shown in Fig. 1. The AlInSb metamorphic buffer consisted of a $1 \mu \mathrm{m}$-thick AlSb buffer, 4 repetitions of 40 AlInSb interlayers and a $10 \mu \mathrm{m}$-thick buffer. The growth rate of around $2 \sim 2.3 \AA / \mathrm{s}$ was kept throughout the whole buffer structure. We added an exceptionally thick layer in this buffer design, which is $10 \mu \mathrm{m}$ AlInSb. We have found that the $10 \mu \mathrm{m}$ thick-buffer does reduce further the TDD but the reduction effect saturates with further increase in thickness and becomes less efficient as it is for the first few $\mu$ m's. However the surface morphology becomes 'cleaner' with the $10 \mu \mathrm{m}$ layer, in the sense that the long growth gives more room for stabilizing the competition of hillocks on the surface and the hillocks develops into sufficiently large walls and makes the study of them much easier.

The inverted QW structure grown on top of the AlInSb buffer has $5 \mathrm{Si} \delta$-doping layers, a $40 \mathrm{~nm}$ AlInSb spacer and a 40nm InSb QW. Work to improve the design of this active region structure is still in progress and the structure presented in this paper is only to demonstrate the optimization of surface morphology for such material systems. An Sb cracker with cracking zone temperature at $900{ }^{\circ} \mathrm{C}$ was used during the growth. The flux ratio of $\mathrm{Sb} /$ Group III was kept at about 1.1, calibrated though the transition of GaSb surface reconstruction using reflection high-energy electron diffraction (RHEED). RHEED was also used to monitor the surface condition of the wafer during the growth. The group III fluxes were measured using beam flux monitor (BFM) (aka monitoring ion gauge (MIG)) before and after the growths, to ensure the desirable (ISP). [19] The AlSb layer, the AlInSb layers and the QW region were grown at a substrate temperature of $500^{\circ} \mathrm{C}, 420^{\circ} \mathrm{C}$ and $380^{\circ} \mathrm{C}$ respectively. More details of growth procedures can be found in Ref. [14]. 


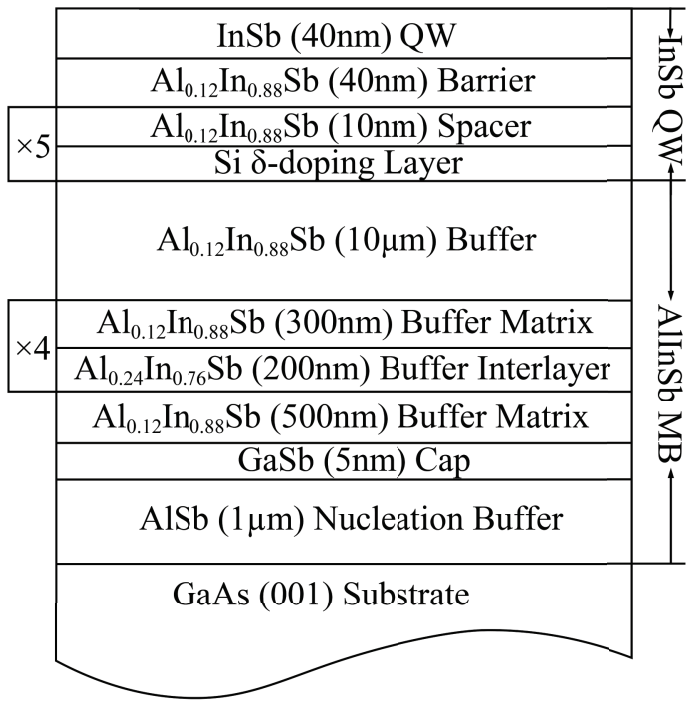

Figure 1: The structure of InSb QW and AlInSb metamorphic buffer (MB) on GaAs (001) substrate.

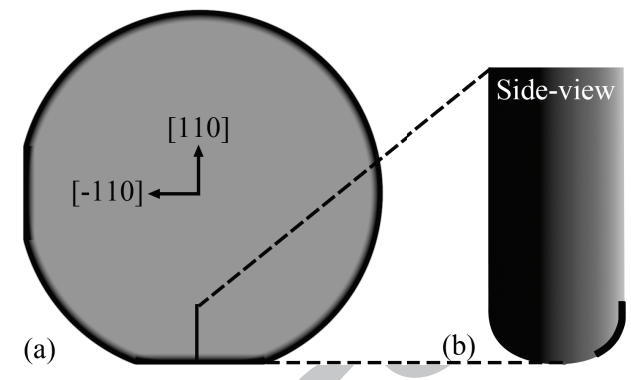

Figure 2: (a) The wafer polishing-induced surface bowing at the edge of the substrate. (b) Cross-section along the solid line in (a). Surface morphology at positions close to the edge on the front polished side of the wafer (region indicated by a black curve) are studied using Nomarski and AFM.

The 2-inch substrate was secured on the 3-inch substrate holder in an unusual suspended set-up with the edge of the wafer completely exposed except for 4 small pins from the holder inserts. Therefore, by employing the polishing-induced gradual change of the surface orientation at the edge of the substrate, as illustrated in Fig. 2, we were able to study the surface morphology AlInSb buffers and InSb QWs at several locations close to the wafer edge as a function of the effective substrate offcut angle.

\section{Results and Discussion}

\subsection{Suppression of Hillocks}

At the wafer centre, a large density of hillocks with spiral atomic-height steps are formed on the surface of the AlInSb metamorphic buffer, as shown in Fig.3(a) and (b). The surface feature of 'dashes' (circled in ovals in Fig.3(a)) is also observed and has been attributed to be micro-twins. [20, 21] By counting the number of atomic steps on hillock sidewalls in $1 \times 1 \mu \mathrm{m}^{2}$ AFM scans in both [110] and [110] directions, we calculated the hillock facet angles to be $0.4 \sim 0.5^{\circ}$ towards [1 10$]$ direction and $0.7 \sim 0.8^{\circ}$ towards [110] direction, similar to what we calculated on the 3 -interlayer buffer without the $10 \mu \mathrm{m}$ thick buffer layer. Close inspections on the hillock sidewalls reveal additional inserted atomic steps (highlighted with a rectangle in Fig. 3(c)), a typical feature of a TD outcrop. The TDs emerging on the hillock sidewalls don't wind up into hillocks and a perfect step-flow growth mode is achieved locally. This provides the most direct hint that the hillock facet angles are the optimum substrate offcut to achieve a hillock-free surface, as what we proposed earlier. [14]

At the very edge of the wafer, as the effective offcut increases towards the edge induced by mechanical polishing, the hillock-dominated surface morphology gradually transitions into a smooth and hillock-free morphology, and eventually to a rough surface with step-bunching and depressed regions (Fig. 4) similarly to what was shown in our earlier work for AlSb. [14] The effective substrate offcut angle was calculated for each inspection site, as shown in Fig. 4. We found that the optimal offcut for the successful suppression of 
hillocks indeed coincide with the facet angles of the hillocks at the centre of the wafer for both [1110] and [110] directions, thus validating our earlier postulate. [14]
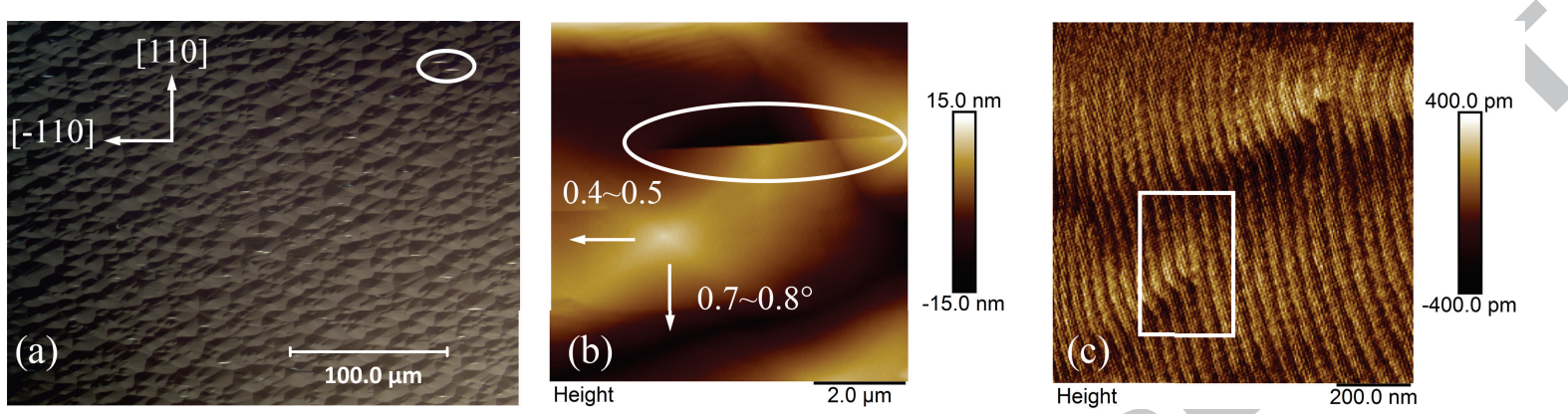

Figure 3: (a) The Surface morphology of the AlInSb metamorphic buffer under Nomarski. (b) An $8 \times 8 \mu m^{2}$ AFM image showing one hillock on the AlInSb buffer surface. The white ovals in (a) and (b) show examples of micro-twins. (c) A $1 \times 1 \mu \mathrm{m}^{2} \mathrm{AFM}$ image showing the sidewall of a hillock with atomic steps in [110] direction and step-insertions (with one example indicated by a white box).
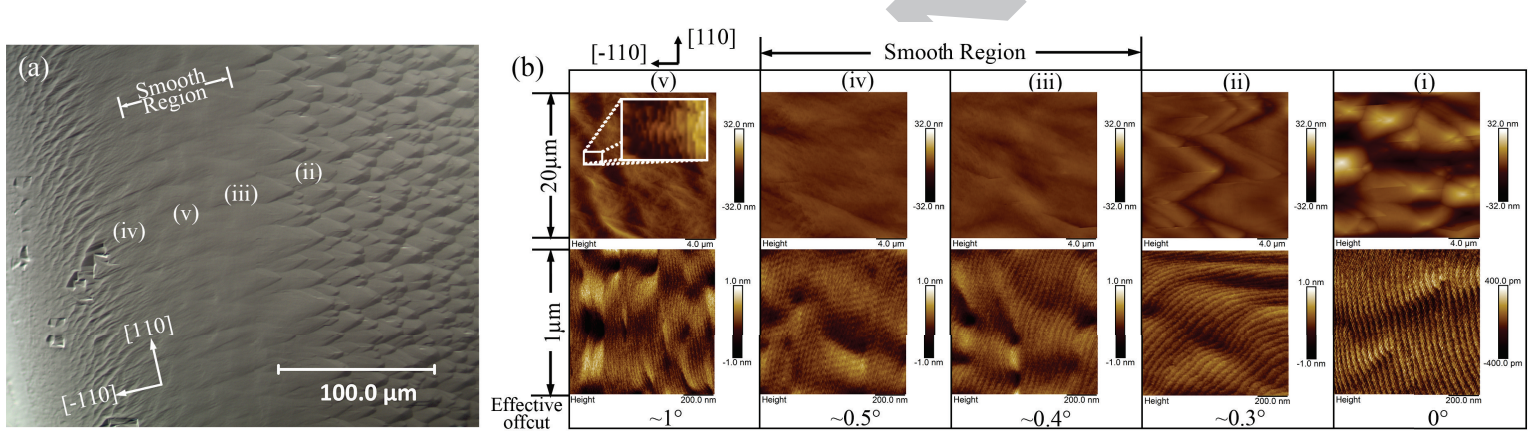

Figure 4: (a) Nomarski and (b) AFM images of $20 \times 20 \mu \mathrm{m}^{2}$ and $1 \times 1 \mu \mathrm{m}^{2}$ in size showing the transition of the AlInSb surface morphology as a function of the effective substrate offcut angle at the edge of the wafer. The approximate AFM scan positions in (b) are indicated by label (ii)-(v) in (a) correspondingly. The effective offcut are calculated using the $1 \times 1 \mu \mathrm{m}^{2}$ AFM images. The insert in (b) panel (v) is a magnified view to show the step-bunching effect.

Now we discuss our observed morphological transitions as a function of substrate offcut, with a graphic illustration in Fig. 5. On the substrate with no offcut, surface adatoms preferentially attach to the atomic steps pinned at TD outcrops and wind up to form pyramid-like hillocks on the surface. As mentioned earlier that adding an additional $10 \mu \mathrm{m}$ to the AlInSb metamorphic buffer doesn't change the facet angle of the hillocks on the surface - the hillocks simply develops in size. Moreover, in an independent experiment with AlSb buffer, we observed only a very weak tend of hillock terraces widening with increasing substrate temperature and/or decreasing growth rate within a broad range of variations in growth conditions. [14] This implies that there exist a natural facet angle for the surface hillocks and the hillocks would grow indefinitely with small hillocks annihilating on the sidewalls of larger hillocks. A symmetric diffusivity for adatoms traversing the step edges in both [1110] and [110] directions was considered for the development of hillocks in the BCF model, which might not be the case. Once the hillocks have developed, the adatom diffusion is 95 influenced primarily by the local surface slope, not by the spiral nature of the entire hillock. Therefore the effect of local uphill and downhill surface currents on the hillock sidewalls should be carefully considered. When substrate offcut is non-zero but less than the natural hillock facet angle, because of the crystalline 
symmetries, the hillocks appear tilted on the surface, as if part of the hillocks is 'buried' by the effective extra surface steps originated from the substrate offcut. When the offcut of the substrate equals exactly to the natural hillock facet angle, the local equilibrium achieved on the hillock sidewalls is now realized globally. Step-flow growth takes place by following the native steps on the substrates and no hillocks can develop. Furthermore, we observed that such step-flow growth can also be achieved with a very narrow range of substrate offcut slightly larger than the natural hillock facet angle. However, when the offcut of the substrate increases further, the growth mode transitions from step-flow to step-bunching. This can be a result of the transition of Ehrlich-Schwoebel barrier from negative to positive, promoting the uphill adatom current and in consequence leading to surface roughening. Moreover, it has been suggested that the surface depressions may form at TD outcrops due to the balance of dislocation-associated strain energy density by surface tension. [22] This effect might also promote the surface roughening process and the surface depressions we observe on the $1 \times 1 \mu \mathrm{m}^{2}$ AFM image in Fig. 4(b) panel (v) might be related to it. Finally, we cannot at this point exclude the possibility that the roughening is related to inferior surface epi-ready preparation when approaching the wafer edge.

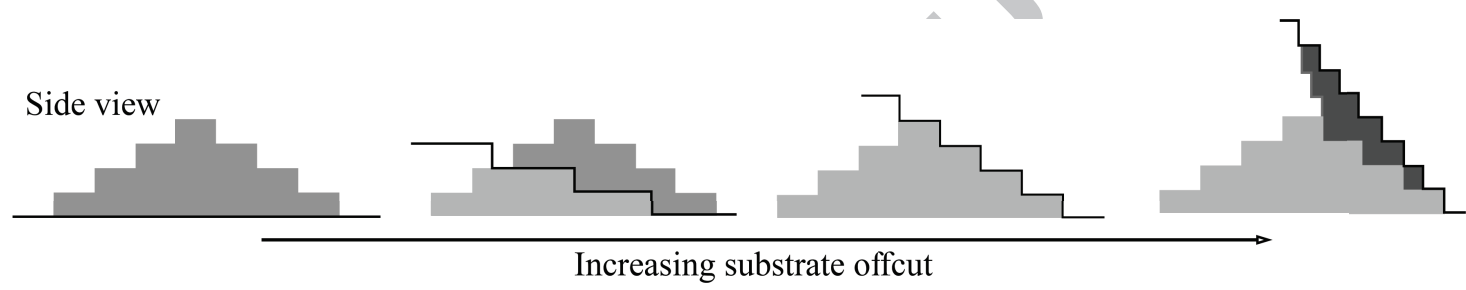

Figure 5: Graphic illustration of the growth mechanism that results in a morphological transition with increasing substrate offcut angle. The black curves are surfaces parallel to the substrate. The medium gray regions represents the hillocks formed on the surface. The light gray and dark gray regions are the 'suppressed parts' and the 'over-suppressed parts' of the hillocks, respectively. Hillock-free and smooth surface can be achieved when the substrate offcut angle agrees with the hillock sidewall angle.

\subsection{Atomically Smooth InSb QWs}

With InSb QW overgrown on the metamorphic buffer, the surface morphology is essentially preserved - a large density of hillocks covering the surface (Fig. 6(a)). However, now two groups of hillocks with different sizes can be easily distinguished. The large hillocks originate from the very thick AlInSb buffer. The small hillocks with slightly steeper sidewalls, which emerge on the top and the sidewalls of the large AlInSb hillocks, are formed from the InSb QW layer at TD outcrops (Fig.2(b)). The two groups of the hillocks provide us an insight into the structure of InSb quantum wells grown on typical metamorphic buffers. As shown in the insertion of Fig.6(a), the quantum well is thicker at the InSb hillocks and thinner elsewhere, which will result in undesirable localization effects. On the other hand, the emergence of of such little InSb hillocks shows that the natural facet angle for InSb is slightly larger than that for AlInSb and a larger substrate offcut is needed for the complete suppression of InSb hillocks. Indeed, at the edge of the InSb QW wafer towards [110] direction, we observed a new morphological transition region where the large AlInSb hillocks are already suppressed while small InSb hillocks still persist. Our calculation of the atomic step density on the $1 \times 1 \mu \mathrm{m}^{2}$ AFM scans confirmed that effective substrate offcut angle for this region is around $0.4 \sim 0.5^{\circ}$, which is the optimum substrate offcut for AlInSb. A hillock-free and atomically smooth InSb surface is found at a slightly steeper substrate offcut angle of around $0.5 \sim 0.6^{\circ}$.

We subsequently regrown the same InSb QW structure on GaAs (001) substrates with a specified offcut of $0.55 \pm 0.02^{\circ}$ towards [1110] direction. A hillock-free and atomically smooth surface morphology is observed on the entire 2-inch wafer (Fig. 8(a) and (b)). By comparing to the same QW structure grown 

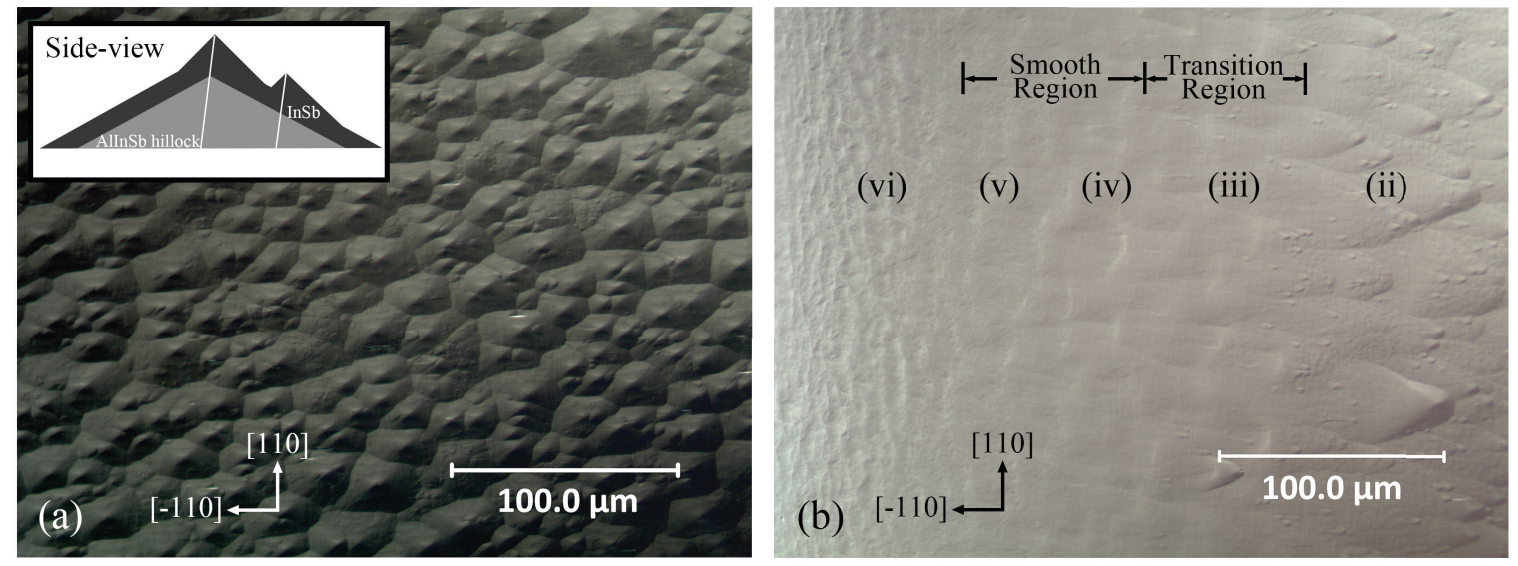

Figure 6: The Surface morphology of the InSb QW under Nomarski at the centre (a) and at the edge (b) of the wafer. The insert in (a) shows a dramatic illustration of the side-view of InSb QW structure. Label (ii)-(vi) in (b) corresponds to the approximate positions of the AFM scans in Fig. 7.

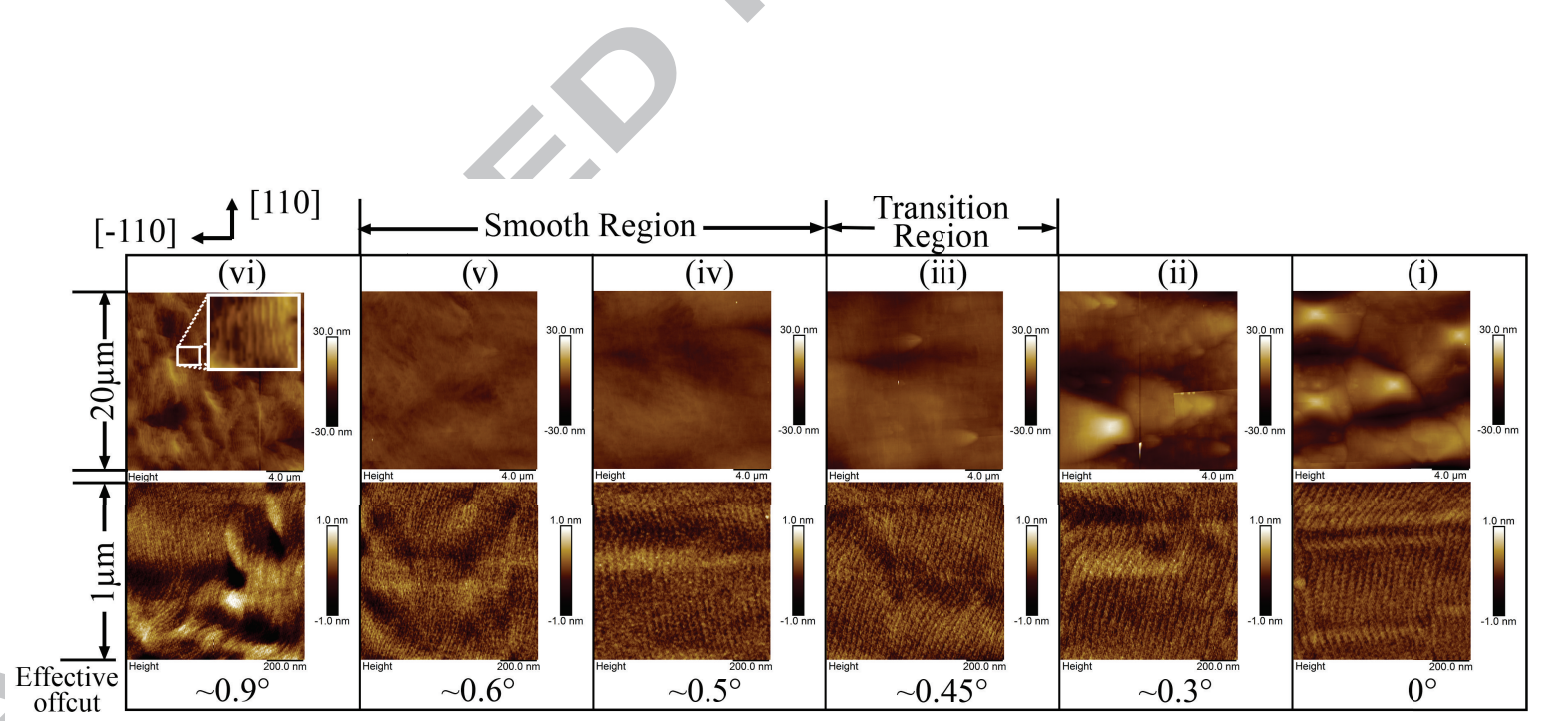

Figure 7: $20 \times 20 \mu \mathrm{m}^{2}$ and $1 \times 1 \mu \mathrm{m}^{2}$ AFM images showing the transition of the InSb surface morphology as a function of effective substrate offcut angle. The insert in panel (vi) is a magnified view to show the step-bunching effect. The approximate AFM scan positions are indicated by label (ii)-(vi) in Fig. 6(a) correspondingly. 
on the on-orientation GaAs (001) substrate in the smooth region at the edge of the wafer, the offcut wafer shows a comparable but even smoother morphology, probably due to the higher substrate quality and more uniform growth conditions at the centre of the wafer. Importantly, we no longer observe any micro-twins on the surface. Micro-twins are more disruptive crystallographic defects than TDs. Though being of a low density, they could have much larger influence on the electric mobility of the InSb QW. The suppression of micro-twins with offcut substrates have been reported [23] and the reason for that is still under investigation. In $1 \times 1 \mu \mathrm{m}^{2}$ AFM scans (Fig. 8(b)), we observed a near-parallel step-flow growth mode and the substrate offcut was calculated to be indeed around $0.5^{\circ} \sim 0.6^{\circ}$. At the edge of the wafer when the offcut just start to increase to around $0.7^{\circ} \sim 0.8^{\circ}$, the offcut-induced rough surface morphology with step-bunching and depressions is again observed (Fig. 8(c)). The fact that the same surface morphology is observed at a location much closer to the centre on the offcut substrate than on the on-orientation substrate supports the argument that such surface roughness is related to the growth kinetics rather than inferior substrate preparation at the wafer edge.

(a)

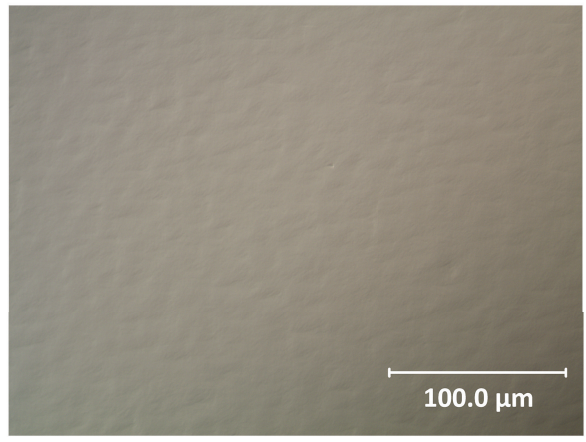

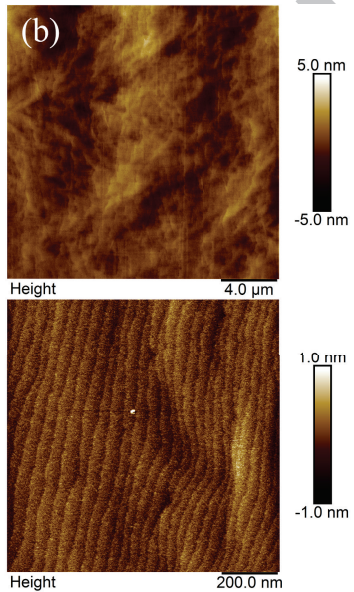

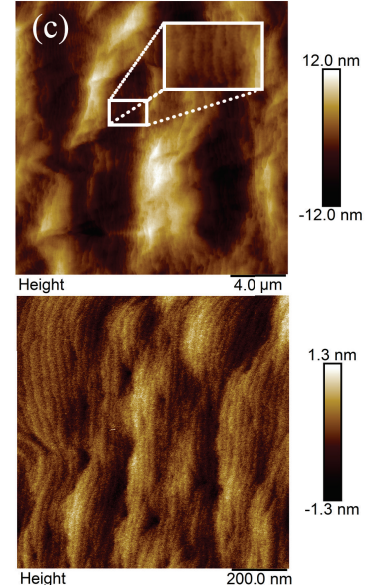

Figure 8: The surface morphology of InSb QW grown on the GaAs offcut substrate observed with (a) Nomarski microscope at the centre of the wafer and $20 \times 20 \mu \mathrm{m}^{2}$ and $1 \times 1 \mu \mathrm{m}^{2}$ AFM scans both (b) at the centre and (c) at the edge of the wafer. The insert in (c) is a magnified view to show the step-bunching effect, similar to that in Fig. 4(b) panel (v) and Fig. 7 panel (vi).

\section{Conclusion}

In summary, we have studied the influence of substrate offcut angle on the morphology of AlInSb metamorphic buffers and atop InSb quantum wells grown on GaAs (001) substrate by MBE. As the effective substrate offcut increases towards the very edge of the wafer due to mechanical polishing, the surface morphology evolves from one which is hillock-dominated to a hillock-free morphology and then transitions into step-bunching and surface depressions. A toy model was discussed to understand the offcut-dependent morphological transitions. We found that hillock-free morphology can be achieved for offcut angle equal to the facet angle of hillocks, which confirmed our postulate in an earlier work [14]. The natural facet angles of hillocks are different for different materials and it may depend weakly on the growth conditions. With our growth conditions, the optimum substrate offcut was found to be around $0.4^{\circ} \sim 0.5^{\circ}$ in [1 110$]$ direction and $0.7 \sim 0.8^{\circ}$ in [110] for AlInSb. The optimum substrate offcut in [110] direction for InSb is $0.5^{\circ} \sim 0.6^{\circ}$, slightly larger than that for AlInSb. Such offcut is effective in suppressing the formation of micro-twins 
as well. On GaAs (001) substrates with the offcut angle optimized for InSb, we have successfully grown hillock-free, micro-twin-free and atomically smooth InSb QWs. Our next step is to grow modulation doped QW structures simultaneously on both the on-orientation and offcut substrates. The mobilities measurements of the two should provide the most direct information on the postulated [18] scattering effect from the hillocks.

\section{Acknowledgments}

The authors acknowledge the support from the Natural Sciences and Engineering Research Council (NSERC) of Canada, the Waterloo Institute of Nanotechnology (WIN) and the Institute of Quantum Computing (IQC). We'd also like to thank Prof. Pu Chen and Prof. Shirley Tang of the University of Waterloo for providing access to their AFM machines.

\section{References}

[1] J. Alicea, Majorana fermions in a tunable semiconductor device, Physical Review B 81 (12) (2010) 125318. doi:10.1103/ PhysRevB.81.125318.

[2] R. M. Lutchyn, J. D. Sau, S. Das Sarma, Majorana fermions and a topological phase transition in semiconductorsuperconductor heterostructures, Physical Review Letters 105 (7) (2010) 077001. doi:10.1103/PhysRevLett.105. 077001.

[3] Y. Oreg, G. Refael, F. von Oppen, Helical liquids and majorana bound states in quantum wires, Physical Review Letters 105 (17) (2010) 177002. doi:10.1103/PhysRevLett.105.177002.

[4] R. M. Lutchyn, E. P. A. M. Bakkers, L. P. Kouwenhoven, P. Krogstrup, C. M. Marcus, Y. Oreg, Majorana zero modes in superconductor-semiconductor heterostructures, Nature Reviews Materials 3 (5) (2018) 52-68. doi:10.1038/ s41578-018-0003-1.

[5] V. Mourik, K. Zuo, S. M. Frolov, S. R. Plissard, E. P. A. M. Bakkers, L. P. Kouwenhoven, Signatures of majorana fermions in hybrid superconductor-semiconductor nanowire devices, Science 336 (6084) (2012) 1003-1007. doi:10.1126/science. 1222360.

[6] L. P. Rokhinson, X. Liu, J. K. Furdyna, The fractional a.c. josephson effect in a semiconductor-superconductor nanowire as a signature of Majorana particles, Nat Phys 8 (11) (2012) 795-799. doi:10.1038/nphys2429.

[7] M. T. Deng, C. L. Yu, G. Y. Huang, M. Larsson, P. Caroff, H. Q. Xu, Anomalous zero-bias conductance peak in a Nb-InSb nanowire-Nb hybrid device, Nano Letters 12 (12) (2012) 6414-6419. doi : 10.1021/nl303758w.

[8] n. Gl, H. Zhang, J. D. S. Bommer, M. W. A. de Moor, D. Car, S. R. Plissard, E. P. A. M. Bakkers, A. Geresdi, K. Watanabe, T. Taniguchi, L. P. Kouwenhoven, Ballistic Majorana nanowire devices, Nature Nanotechnology 13 (3) (2018) $192-197$. doi:10.1038/s41565-017-0032-8.

[9] H. J. Suominen, M. Kjaergaard, A. R. Hamilton, J. Shabani, C. J. Palmstrm, C. M. Marcus, F. Nichele, Zero-energy modes from coalescing andreev states in a two-dimensional semiconductor-superconductor hybrid platform, Physical Review Letters 119 (17) (2017) 176805. doi:10.1103/PhysRevLett.119.176805.

[10] T. D. Mishima, M. B. Santos, Effect of buffer layer on InSb quantum wells grown on GaAs (001) substrates, Journal of Vacuum Science \& Technology B 22 (3) (2004) 1472-1474. doi:10.1116/1.1699340.

[11] T. D. Mishima, M. Edirisooriya, N. Goel, M. B. Santos, Dislocation filtering by $\mathrm{Al}_{x} \mathrm{In}_{1-x} \mathrm{Sb} / \mathrm{Al}_{y} \mathrm{In}_{1-y} \mathrm{Sb}$ interfaces for insbbased devices grown on GaAs (001) substrates, Applied Physics Letters 88 (19) (2006) 191908. doi:10.1063/1.2203223.

[12] J. M. S. Orr, A. M. Gilbertson, M. Fearn, O. W. Croad, C. J. Storey, L. Buckle, M. T. Emeny, P. D. Buckle, T. Ashley, Electronic transport in modulation-doped InSb quantum well heterostructures, Physical Review B 77 (16) (2008) 165334. doi:10.1103/PhysRevB.77.165334.

[13] C. A. Lehner, T. Tschirky, T. Ihn, W. Dietsche, J. Keller, S. Flt, W. Wegscheider, Limiting scattering processes in highmobility InSb quantum wells grown on GaSb buffer systems, Physical Review Materials 2 (5) (2018) 054601. doi: 10. 1103/PhysRevMaterials. 2.054601.

200 [14] Y. Shi, D. Gosselink, K. Gharavi, J. Baugh, Z. R. Wasilewski, Optimization of metamorphic buffers for MBE growth of high quality AlInSb/InSb quantum structures: Suppression of hillock formation, Journal of Crystal Growth 477 (Supplement C) (2017) 7-11. doi:10.1016/j.jcrysgro.2017.03.043.

[15] W. K. Burton, N. Cabrera, F. Frank, The growth of crystals and the equilibrium structure of the their surfaces, Philosophical Transactions of the Royal Society of London Series a Mathematical Physical and Engineering Sciences 243 (1951) 299-358. doi:10.1098/rsta.1951.0006. 
[16] H. Turski, M. Siekacz, M. Sawicka, G. Cywinski, M. Krysko, S. Grzanka, J. Smalc-Koziorowska, I. Grzegory, S. Porowski, Z. R. Wasilewski, C. Skierbiszewski, Growth mechanism of InGaN by plasma assisted molecular beam epitaxy, Journal of Vacuum Science \& Technology B 29 (3) (2011) 03C136 - 03C136-5. doi:10.1116/1.3590932.

[17] C. Skierbiszewski, H. Turski, G. Muziol, M. Siekacz, M. Sawicka, G. Cywinski, Z. R. Wasilewski, S. Porowski, Nitridebased laser diodes grown by plasma-assisted molecular beam epitaxy, Journal of Physics D-Applied Physics 47 (7) (2014) 18. doi:10.1088/0022-3727/47/7/073001.

[18] C. McIndo, D. Hayes, A. Papageorgiou, L. A. Hanks, G. Smith, C. Allford, S. Zhang, E. M. Clarke, P. Buckle, Optical microscopy as a probe of the rate limiting transport lifetime in $\mathrm{InSb} / \mathrm{Al}_{1-x} \mathrm{In}_{x} \mathrm{Sb}$ quantum wells, Journal of Physics: Conference Series 964 (1) (2018) 012005. doi:10.1088/1742-6596/964/1/012005.

[19] M. C. Tam, Y. Shi, D. Gosselink, M. Jaikissoon, Z. R. Wasilewski, Temperature monitoring of narrow bandgap semiconductors, Journal of Vacuum Science \& Technology B, Nanotechnology and Microelectronics: Materials, Processing, Measurement, and Phenomena 35 (2) (2017) 02B102. doi : 10.1116/1.4975926.

[20] T. D. Mishima, J. C. Keay, N. Goel, M. A. Ball, S. J. Chung, M. B. Johnson, M. B. Santos, Anisotropic structural and electronic properties of $\mathrm{InSb} / \mathrm{Al}_{x} \mathrm{In}_{1-x} \mathrm{Sb}$ quantum wells grown on GaAs $\left(\begin{array}{lll}0 & 0 & 1\end{array}\right)$ substrates, Journal of Crystal Growth 251 (14) (2003) 551-555. doi : 10.1016/S0022-0248(02) 02183-8.

[21] T. D. Mishima, J. C. Keay, N. Goel, M. A. Ball, S. J. Chung, M. B. Johnson, M. B. Santos, Effect of structural defects on $\mathrm{InSb} / \mathrm{Al}_{x} \mathrm{In}_{1-x} \mathrm{Sb}$ quantum wells grown on GaAs (001) substrates, Physica E: Low-dimensional Systems and Nanostructures 20 (34) (2004) 260-263. doi:10.1016/j . physe. 2003.08.014.

[22] F. Frank, Capillary equilibria of dislocated crystals, Acta Crystallographica 4 (6) (1951) 497-501. doi:10.1107/ S0365110X51001690.

[23] T. D. Mishima, M. Edirisooriya, M. B. Santos, Reduction of microtwin defects for high-electron-mobility InSb quantum wells, Applied Physics Letters 91 (6) (2007) 062106. doi:10.1063/1.2768033. 


\section{Highlights:}

- Surface morphology of InSb QWs was studied as a function of GaAs substrate offcut.

- Hillock-free morphology was achieved when substrate offcut matches hillock facet.

- Atomically smooth morphology was achieved for the entire wafer of InSb QWs. 